\title{
Overlap Between Computed Tomographic Features Of Hemorrhagic Cerebral Venous Thrombosis And Amyloid Angiopathy
}

K Afifi, Florey Institute of Neuroscience and Mental Health, Melbourne ,Australia and Menoufia University, Alminufya Egypt; PJ Buyck, R Lemmens, P Demaerel, University Hospitalis Leuven ,Belgium; SM Zuurbier, JM Coutinho, Amsterdam Univ Medical centers, Amsterdam, The Netherlands; CG Esperon, John Hunter Hospital, Newcastle,Australia; MA Barboza and A Arauz, , Instituto Nacional de Neurología y Neurocirugía Dr. Manuel Velasco Suárez, México City, México; P Costa, University of Brescia, Italy; I Escudero, J Montaner, University Hospital Virgen del Rocio, Sevilla, Spain; A Pezzini, University of Brescia, Italy; C Weimar, University Hospital Essen, Essen, Germany; M Schlamann, University of Cologne, Germany; D Renard, Nîmes University Hospital, France; F Fazekas ,T Gattringer, N Hinteregger, Medical University of Graz, Austria; E Giralt-Steinhauer, J Jimenez Conde, Hospital del Mar, Barcelona, Spain; J Putaala, S Hiltunen, Helsinki University Hospital, Helsinki, Finland; T Tatlisumak, University of Gothenburg, Gothenburg, Sweden, V Thijs, Florey Institute of Neuroscience and Mental Health, University of Melbourne, Melbourne ,Australia.

\section{Background}

-Amyloid angiopathy and cerebral venous thrombosis lead to lobar hemorrhage and subarachnoid hemorrhage.

-Given the relatively high prevalence of amyloid angiopathy in the elderly population, incidental cooccurrence of these conditions is not surprising.

\section{Objectives}

- To study the overlap between these two conditions.

- We study the prevalence of common imaging markers of cerebral amyloid angiopathy in a large cohort of patients with established hemorrhagic cerebral venous thrombosis.

\section{Methods}

- Edinburgh CT criteria and the modified Boston criteria for cerebral amyloid angiopathy were applied by 2 independent researchers to patients with confirmed diagnosis of hemorrhagic cerebral venous thrombosis.

- We identified lobar hemorrhage with or without finger like projections, subarachnoid hemorrhage, microbleeds \& cortical superficial siderosis.

- Neither histopathological study of the hematoma nor cortical biopsy of our cases was done.

- APOE genotyping was not available
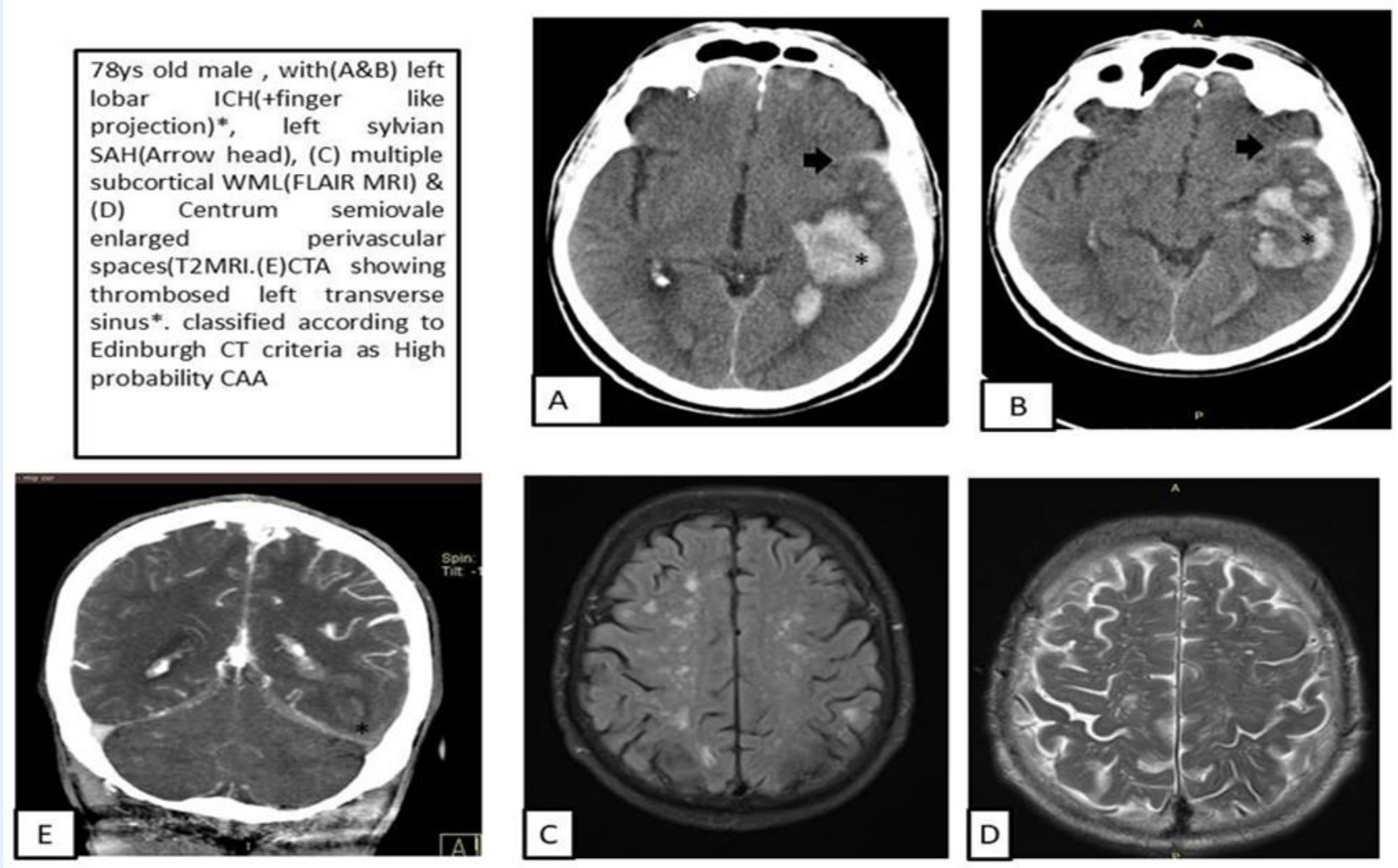

\section{Results}

- Study Cohort:102 patients,10 institutions

- Mean age :46 Ys (73\% females)
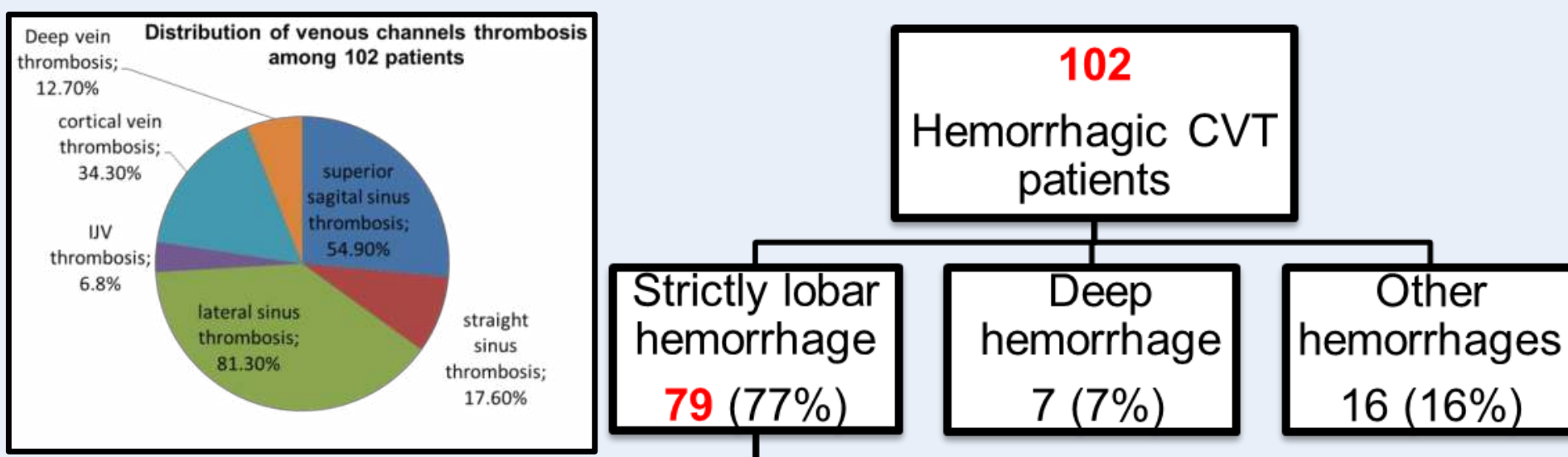

Combined lobar hemorrhage \& SAH $20 / 79$

Lobar hemorrhage with finger like projections

$40 / 79$
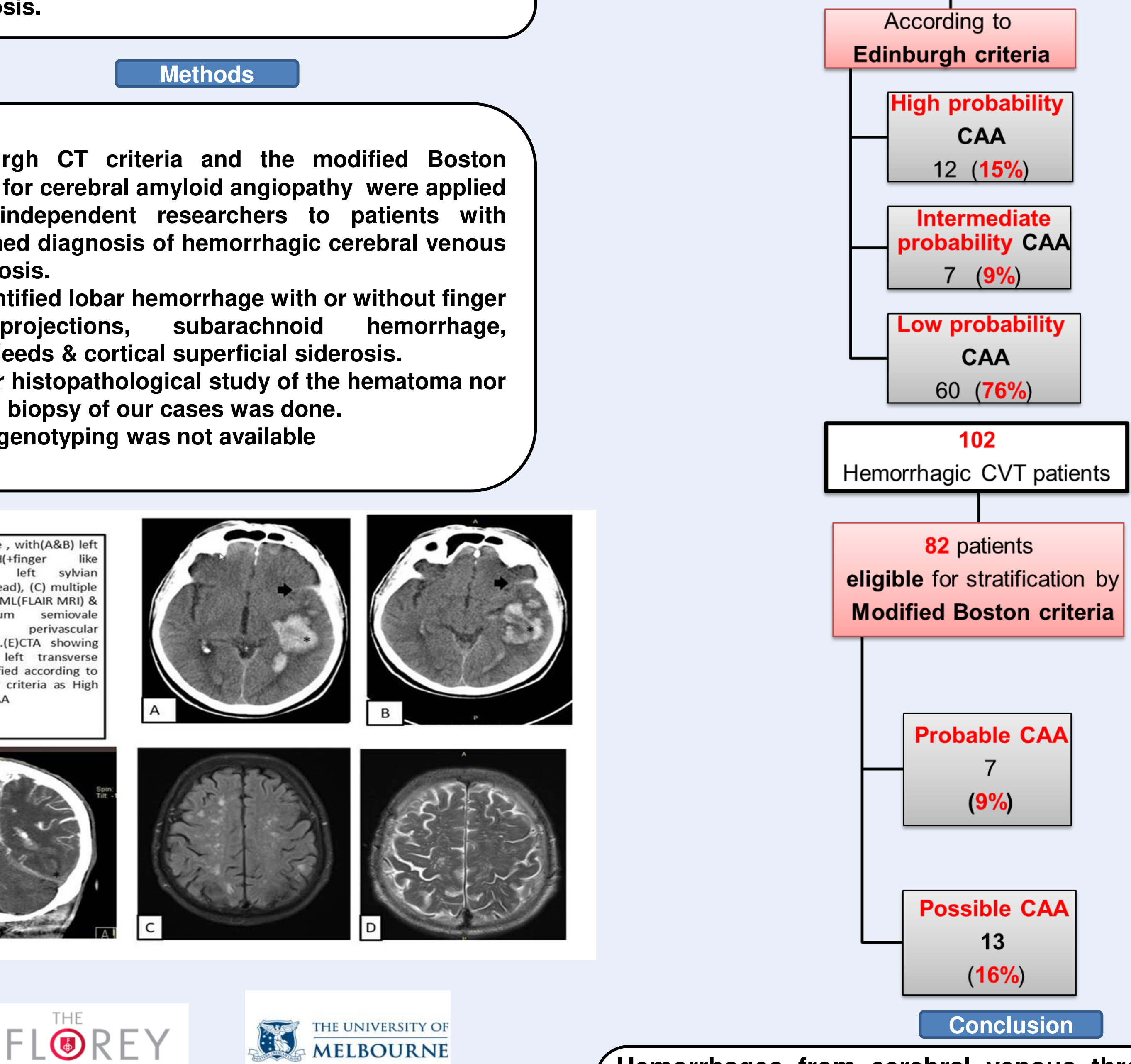

Conclusion

Hemorrhages from cerebral venous thrombosis may mimic cerebral amyloid angiopathy related hemorrhages. Therefore, the presence of cerebral venous thrombosis needs to be considered in patients who present with hemorrhage suggestive of cerebral amyloid angiopathy 